\title{
Revisit of fading channel characteristics in IEEE 802.11 WLANs: independent and burst transmission errors
}

\author{
P. Chatzimisios ${ }^{1}$, V. Vitsas ${ }^{1}$ and A. C. Boucouvalas ${ }^{2}$ \\ ${ }^{1}$ Department of Informatics, \\ Technological Educational Institution, Thessaloniki, Greece, \\ pchatzimisios@ieee.org,vitsas@it.teithe.gr \\ ${ }^{2}$ Multimedia Communications Research Group, School of Design, Engineering and Computing, \\ Bournemouth University, Fern Barrow, Poole, UK, \\ tboucouv@bournemouth.ac.uk
}

\begin{abstract}
IEEE 802.11 is worldwide implemented being the most widely deployed protocol for Wireless Local Area Networks (WLANs). In a realistic environment, WLANs encounter channel fading which causes independent and burst transmission errors which in addition with packet collisions degrade overall performance. In this paper, we present a simple and effective contention window-resetting scheme, named Double Increment Double Decrement (DIDD), to improve the performance of the Binary Exponential Backoff (BEB) scheme utilized in IEEE 802.11. Our work becomes important and meaningful since it analyzes and models the error patterns in order to evaluate their potential impact on throughput efficiency and average packet delay performance. In particular, we provide useful insights by exploring the effect of independent and burst transmission errors, packet size and network size on the performance of the DIDD scheme.
\end{abstract}

\section{Introduction}

In recent years, the widespread use of multimedia applications has created new requirements upon the underlying Wireless LANs (WLANs) and brought to the foreground the IEEE $802.11 \mathrm{a} / \mathrm{b} / \mathrm{g}$ standards [1]. The 802.11 protocol of the Institute of Electrical and Electronics Engineers (IEEE) is the most popular standard for WLANs and specifies both the Medium Access Control (MAC) and the Physical (PHY) Layers [1]. The PHY layer selects the best modulation scheme given the channel conditions, whereas the MAC layer makes the decision by utilizing a distributed manner on how the offered bandwidth is shard among all stations. The fundamental MAC mechanism is the Distributed Coordination Function (DCF), which implements the Carrier Sense Multiple Access with Collision Avoidance (CSMA/CA) method.

\section{A. Related work}

Numerous research efforts have been conducted on modelling the behaviour [2]-[6] as well as improving the performance of IEEE 802.11 DCF [7]-[17]. The bidimensional Markov chain modeling, first introduced by
Bianchi in [2], has become the most common method for calculating the saturated performance of the IEEE 802.11 protocol. Wu in [3] has built on this model and presented a mathematical analysis that takes into account packet retry limits. In [4], we developed a new performance analysis based on the Markov chain model of [3] that allowed the calculation of the average packet delay and other performance metrics for IEEE 802.11 DCF. Vukovic in [5] extended Bianchi's and our previous work by developing a simpler one-dimensional Markov chain model but without proposing any protocol enhancement. Cali in [6] attempted to approach protocol capacity by replacing the exponential backoff mechanism with an adaptive one but under the assumption that the backoff time is sampled from a geometric distribution. Carvalho in [7] considered the impact of the minimum Contention Window $(\mathrm{CW})$ size but not combined with any other protocol parameters. Work in [8] and [9] utilizes a different modelling approach of IEEE 802.11 DCF by employing elementary conditional probability arguments rather than bi-dimensional Markov chains.

A lot of research has been conducted on improving the performance of IEEE 802.11 DCF by utilizing many different approaches. In [10], we have extended the mathematical model of [4] by considering packet bursting, a technique in which a station transmits more than one data packets when it gets hold of the medium and, thus, improves considerably protocol performance. Work in [8] and [11] has studied the effectiveness of the RTS/CTS reservation scheme in reducing collision duration for high data rates and an all-purpose expression was derived for the optimal use of the RTS/CTS handshake aiming to maximize performance. In [12], we have studied an appropriate tuning of the backoff algorithm by proposing three sets of parameter values for initial contention window size, retry limit and number of backoff stages in order to achieve better performance on particular metrics for specific communication needs. Authors in [13]-[15] also suggested certain modifications of the backoff scheme but either their work is based only on simulation [13]-[14] or they do not study at all packet delay performance [15].

Most of the research work presented in the literature 
The 17th Annual IEEE International Symposium on Personal, Indoor and Mobile Radio Communications (PIMRC'06)

assumes error-free transmissions. Recently, several papers consider the impact of error-prone channels on the IEEE 802.11 performance. More specifically, Crow in [16] first studied the effect of errors on performance by means of simulation. Authors in [17] and [18] also considered transmission errors by means of a Markov chain model but investigated only saturation throughput. Latest work in [19] and [20] studies error-prone environments but only focuses in the effect of retry limits on the IEEE 802.11 performance. In [21] we have proposed an easy-to-implement backoff algorithm named DIDD (Double Increment Double Decrement) that gradually decreases the Contention Window $(C W)$ after a successful packet transmission.

\section{B. Overview}

The main limitation of all these approaches is that little insight is provided into the nature of errors or they do not consider burst errors (the analysis of bursty behavior is not straightforward but rather complex requiring a careful study). However, the assumption of an ideal error-free wireless channel is not always true in a realistic environment and performance may be overestimated.

In this paper, we will re-examine certain previous errorfree performance results in the literature under the light of realistic link error rate conditions. Our results take into account all the protocol parameters and packet overheads introduced by both the Medium Access Control (MAC) and the physical (PHY) layers as specified in IEEE 802.11b. More specifically, we evaluate the impact of an error-prone channel on unsuccessful transmission probability and its impact on the overall performance of the proposed DIDD scheme in terms of throughput efficiency and average packet delay. Finally, the nature of transmission errors is analyzed and is further categorized to independent with fixed Bit Error Rate $(B E R)$ as well as to time-variable burst errors modelled by the widely used two-state Gilbert-Elliot (GE) Markov chain model ${ }^{1}[22]$.

\section{An overview of IEEE 802.11 BEB and DIDD}

The current section briefly presents the fundamental features and outlines the differences between the Binary Exponential Backoff (BEB) utilized in IEEE 802.11 and DIDD backoff schemes. The channel contention procedures of the legacy DCF define that a station with a packet to transmit first senses the medium activity to ascertain whether it is in use. If the medium is sensed to be idle for a time interval greater than the Distributed Inter-Frame Space (DIFS), the station initiates a packet transmission by transmitting the data packet in basic access. If the medium is sensed busy, the station defers transmission and initializes its random backoff timer. The backoff timer is decremented when the medium is idle, is frozen when the medium is sensed busy and resumes again only after the medium has been idle for longer than a DIFS time interval. Each station is allowed to transmit only when its backoff timer reaches zero.

\footnotetext{
${ }^{1}$ Although the considered GE model does not provide accurate results under any scenario [23], it can serve as a simple and insightful alternative approach to model burst transmission errors.

$1-4244-0330-8 / 06 / \$ 20.00 @ 2006$ IEEE
}

According to the collision avoidance procedures of $\mathrm{BEB}$, the value of the backoff timer value for each station is uniformly chosen in the interval $\left[0, W_{i}-1\right]$, where $W_{i}$ is the current contention window $(C W)$ size, $i$ is the backoff stage, $i \in[0, m]$ and $m$ represents the number of backoff stages. At the first transmission attempt, $C W$ is equal to the minimum backoff window size $W=C W_{\min }$. If the backoff timer of two or more stations expires at the same time, their packet transmissions overlap and a packet collision occurs. Thus, we define as packet collision the event that at least two stations initiate transmission at the same time and the receivers cannot decode any packets correctly. On the other hand, we define as transmission error the event that there is only one station transmitting but the channel is so noisy (caused by channel fading, path loss, thermal noise or interferences from other radio sources) that the receiver cannot decode the whole packet successfully.

Under BEB, after each unsuccessful transmission due to a packet collision or a transmission error, $W_{i}$ is doubled until a maximum value is reached denoted by $C W_{\max }$. Once it reaches $C W_{\max }, W_{i}$ will remain at this value until it is reset. The success of a transmission is inferred from the reception of a positive acknowledgment (ACK) packet from the destination station, a Short Inter-Frame Space (SIFS) interval after the successful reception of a data packet. After receiving the ACK packet, the source station resets $C W$ to $C W_{\min }$ and contends again for medium access if a packet is available. If the source station does not receive an $\mathrm{ACK}$, the data packet is assumed to have been lost and a retransmission is scheduled by doubling the $\mathrm{CW}$ according to the specified backoff procedures. Ideally, the $C W$ size should be increased only when packet losses are due to collisions in order to decrease congestion, since increasing the $C W \psi$ size in the case of transmission errors may degrade the data throughput and may increase the transmission delays. However, a station cannot distinguish collisions from transmission errors at the MAC layer and it handles them in a same way.

The main difference between the legacy BEB and DIDD is that according to the latter scheme after a successful packet transmission the contention window is halved and not reset to $C W_{\min }$ as in BEB (see figure 1).

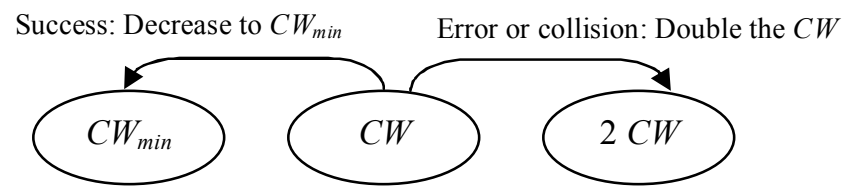

(a) Legacy Binary Exponential Backoff (BEB) scheme

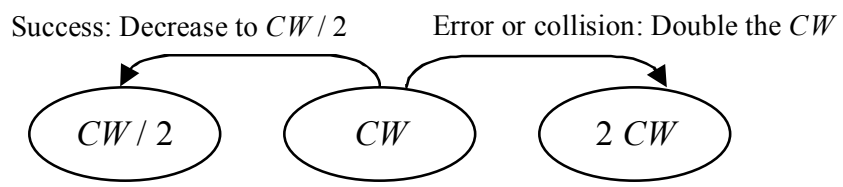

(b) Double Increment Double Decrement (DIDD) backoff scheme

Figure 1 Comparison of $\mathrm{CW}$ process of the two backoff schemes 
The 17th Annual IEEE International Symposium on Personal, Indoor and Mobile Radio Communications (PIMRC'06)

\section{Mathematical Modelling}

\section{A. Analytical framework and assumptions}

We follow closely [8] and [9] by making use of the same assumptions; the network consists of $n$ contending stations, each station has always a packet available for transmission and no hidden stations exist since all the stations can hear each other [24]. Note that the mathematical modeling of the DIDD backoff scheme can be developed by utilizing three different approaches as shown in [8]. In particular, we can either employ a two-dimensional Markov chain model [3], a one-dimensional Markov chain model [5] or elementary conditional probability arguments [8].

The main difference between the proposed model in this paper and the model that assumes no transmission errors (only packet collisions) is that here we define as $p_{f}$ the collision-error probability, which is the probability that a transmitted packet encounters a collision (at least one of the $n-1$ remaining stations transmit in the same time slot) or is received in error due to channel fading and/or noise. Thus, the equations for calculating the transmission probability as well as other performance metrics (such as throughput and packet delay) will be different from the equations computed for an error-free channel in [4] that should be appropriately modified to include transmission errors.

In an error-prone environment, the packet error rate depends on the bit error rate, the packet header and the packet length:

$$
P E R=1-(1-B E R)^{l+h d r}
$$

where $B E R$ is the link bit error rate, $l$ is the packet payload size and $h d r$ is the packet header length.

By following closely and utilizing any of the previously mentioned modeling approaches [3][5][8], we can calculate the probability $\tau$ that a station transmits a packet in a randomly chosen slot time. The two schemes reach different equations for $\tau$ since they employ different contention window adjustment mechanisms as specified by the collision avoidance procedures. For the legacy 802.11 BEB, we have [4]:

$$
\tau=\frac{2\left(1-2 p_{f}\right)\left(1-p_{f}^{m+1}\right)}{W\left(1-\left(2 p_{f}\right)^{m+1}\right)\left(1-p_{f}\right)+\left(1-2 p_{f}\right)\left(1-p_{f}^{m+1}\right)}
$$

For the DIDD scheme, $\tau$ is expressed as in [21]:

$$
\tau=\frac{2(1-2 a)\left(1-a^{m+1}\right)}{\left(1-(2 a)^{m+1}\right)(1-a) W+(1-2 a)\left(1-a^{m+1}\right)}
$$

where $a=p_{f} / 1-p_{f}, m=\log _{2}\left(\frac{C W_{\max }+1}{C W_{\min }+1}\right)$ is the number of backoff stages and $p_{f}$ is the collision-error probability that a transmitted packet encounters a collision (with probability $\left.p_{c o l}\right)$ or is received in error (with probability $P E R$ ). To calculate $p_{f} \psi$, we assume that at each transmission attempt, regardless of the number of retransmissions, each packet has a constant and independent failure probability given $\psi$ by. $\psi$

$$
p_{f}=1-\left(1-p_{c o l}\right)(1-P E R)=1-(1-\tau)^{n-1}(1-B E R)^{l+h d r}
$$

Equations (2) (4) and (3) (4) respectively form nonlinear systems with two unknowns $\tau \in(0,1)$ and $p_{f} \in(0,1)$ that can be solved using numerical methods having a unique solution (the interested reader can find a proof of the uniqueness in the Appendix of [12]).

Next, we employ the simple and well known GilbertElliott (GE) model [22] and we work out an accurate estimate of the link BER for the case of burst transmission errors. The wireless channel is modeled as a discrete time Markov chain and is assumed as having two states; the $G O O D$ state (representing the channel under normal conditions) and the $B A D$ state (representing a channel fade). Within each state, bit errors occur independently with rates $B E R_{G}$ and $B E R_{B}$, respectively $\left(B E R_{G}<<B E R_{B}\right)$. This means that bits sent over the wireless channel are facing a certain bit error rate, where an estimate of it is determined by the channel state as:

$$
B E R_{b u r s t}=\frac{B E R_{G} p_{b g}+B E R_{B} p_{g b}}{p_{b g}+p_{g b}}
$$

where $p_{g b}$ and $p_{b g}$ represent the transition probabilities from the $G O O D$ to the $B A D$ state and from the $B A D$ to the $G O O D$ state respectively. The transition probabilities $p_{g b}$ and $p_{b g}$ are related to $T_{B A D}$ and $T_{G O O D}$, the mean sojourn time intervals in the two states i.e. the average time of transmitting bits in $B A D$ (error burst) and GOOD (errorfree burst) states respectively by the following equations:

$$
T_{B A D}=\frac{1}{p_{b g}}=\frac{1}{1-p_{b b}} \text { and } T_{G O O D}=\frac{1}{p_{g b}}=\frac{1}{1-p_{g g}}
$$

\section{B. Saturation Throughput Efficiency}

Following the same reasoning with [2], [3] and [4], we calculate the saturation throughput efficiency $S$ by dividing the time utilized for transmitting payload information in a slot time with the average duration of a slot time $E[$ slot $]$.

In order to compute $E[$ slot $]$, we consider four different types of events taking place in a random chosen slot. Firstly, if no station transmits any packets, all stations wait for duration equal to $T_{\text {idle }}$ that corresponds to the idle slot interval. Secondly, if only one station transmits and its packet transmission is successful, the channel is sensed busy because of a successful transmission for duration equal to $T_{s u c}$. Thirdly, if at least two stations simultaneously transmit then a packet collision occurs and the channel is sensed busy for duration equal to $T_{c o l}$. Finally, if only one station transmits but its packet is corrupted due to channel errors then the channel is sensed busy for duration equal to $T_{\text {err. }}$.

The previously mentioned events $\left(T_{\text {idle }}, T_{\text {suc }}, T_{\text {col }}\right)$ have been extensively studied in [2]-[6]. However, the calculation of $T_{c o l}$ in the literature (i.e., $T_{c o l}=T_{h d r}+T_{D A T A}$ $+D I F S$ ) does not comply with the IEEE 802.11 standard [1]. Actually, $T_{c o l}$ should include the EIFS (Extended IFS interval) and be equal to $T_{h d r}+T_{D A T A}+E I F S=T_{h d r}+T_{D A T A}$ + SIFS $+T_{A C K}+D I F S$ as it has been reported correctly in [9] and [12]. Thus, the duration of the above events that may occur in a randomly chosen slot time can be expressed as: 
The 17th Annual IEEE International Symposium on Personal, Indoor and Mobile Radio Communications (PIMRC'06)

$$
\left\{\begin{array}{l}
T_{i d l e}=\sigma \\
T_{s u c}=T_{D A T A}+S I F S+T_{A C K}+D I F S \\
T_{c o l}=T_{D A T A}+S I F S+T_{A C K}+D I F S \\
T_{e r r}=T_{D A T A}+T_{E I F S} \\
T_{s u c}=T_{c o l}=T_{e r r}
\end{array}\right.
$$

For the case of the DIDD scheme (there are no packet drops at all), $E[X]$ is calculated as $[21]$ :

$$
E[X]=\frac{1}{\tau\left(1-p_{f}\right)}=\frac{1}{\tau(1-\tau)^{n-1}}
$$

In the case of independent transmission errors and by considering the above events, throughput efficiency can be derived as:

$$
S_{i e}=\frac{\left(1-P_{\text {idle }}\right) P_{\text {suc }} l / C}{P_{\text {idle }} \sigma+\left(1-P_{\text {idle }}\right) P_{\text {suc }} T_{\text {suc }}+\left(1-P_{\text {idle }}\right) P_{\text {col }} T_{\text {col }}+\left(1-P_{\text {idle }}\right) P_{\text {err }} T_{\text {err }}}
$$

where the denominator of equation $(8)$ denotes the average length of a slot time $E[$ slot $], T_{\text {idle }}, T_{\text {suc }}, T_{\text {col }}$ and $T_{\text {err }}$ can be obtained from equation (7), $C$ is the data rate and $P_{\text {idle }}, P_{\text {suc }}$, $P_{c o l}, P_{\text {err }}$ are the corresponding probabilities for the above events that we will calculate next.

Let $P_{\text {idle }}$ be the probability that no packet transmission occurs in a randomly chosen slot time and $P_{\text {suc }}$ the probability that an ongoing transmission is successful is obtained when the transmitted packet does not experience either a collision or an error, respectively:

$$
P_{\text {idle }}=(1-\tau)^{n} \quad P_{\text {suc }}=\frac{n \tau(1-\tau)^{n-1}}{1-(1-\tau)^{n}}(1-P E R)
$$

The probability $P_{c o l}$ that an occurring transmission collides because two or more stations simultaneously transmit and the probability $P_{\text {err }}$ that a packet does not experience a collision but is corrupted because of transmission errors are respectively given by:

$$
P_{c o l}=1-\frac{n \tau(1-\tau)^{n-1}}{1-(1-\tau)^{n}} \quad P_{e r r}=\frac{n \tau(1-\tau)^{n-1}}{1-(1-\tau)^{n}} P E R
$$

In the case of burst errors modeled by the Gilbert-Elliott model, a slightly different way to calculate the throughput efficiency is needed. In particular, we calculate throughput efficiency $S_{G O O D}$ for the $G O O D$ state and $S_{B A D}$ for the $B A D$ state by substituting into equation (8) the corresponding expressions for $p_{f}$. Therefore, we have:

$$
S_{\text {burst }}=\frac{S_{G O O D} T_{G O O D}+S_{B A D} T_{B A D}}{T_{G O O D}+T_{B A D}}
$$

\section{Average Packet Delay}

The delay $D$ is defined to be the time interval from the time a packet is at the head of its MAC queue ready for transmission, until an acknowledgement for this packet is received. Thus, the average packet delay $E[D]$ is given by:

$$
E[D]=E[X] E[\text { slot }]
$$

where $E[X]$ is the average number of slot times required for a successful packet transmission.

For the case of the legacy $\mathrm{BEB}, E[X]$ is calculated as [4]:

$$
E[X]=\sum_{i=0}^{m}\left[\frac{W_{i}+1}{2} \frac{\left(p_{f}{ }^{i}-p_{f}{ }^{m+1}\right)}{1-p_{f}{ }^{m+1}}\right]
$$

Note that if a packet is dropped because it has reached the specified retry limit, the time delay for this packet will not be included in the calculation of the average packet delay since this packet is not successfully received.

Equations (12)-(14) are utilized when independent transmission errors are being considered. In the case of burst errors, packet delay is evaluated differently like as in equation (11); we calculate packet delay $E[D]_{G O O D}$ for the $G O O D$ state and $E[D]_{B A D}$ for the $B A D$ state:

$$
E[D]_{\text {burst }}=\frac{E[D]_{G O O D} T_{G O O D}+E[D]_{B A D} T_{B A D}}{T_{G O O D}+T_{B A D}}
$$

\section{Performance Evaluation}

In this section, we only report performance results for the proposed DIDD scheme. A detailed analysis as well as a performance comparison between the legacy BEB and DIDD schemes can be found in [21]. We utilize IEEE $802.11 \mathrm{~b}$ [1] as the underlying PHY and all data and control packets are transmitted at the rate of $C=1 \mathrm{Mbit} / \mathrm{s}$. Although we only consider the basic access scheme, the current work can be easily extended for the RTS/CTS handshaking mechanism as well as for the case of higher data transmission rates of IEEE 802.11b/a/g PHY layers.

The current work considers two different error models; independent errors with a fixed BER and time-variable errors that follow a bursty behavior according to a GE model $\left(B E R_{G}=10^{-10}, B E R_{B}=10^{-5}, \quad T_{G O O D}=33.333\right.$ and $\left.T_{B A D}=10\right)$.

Figure 2 illustrates the error-collision probability $p_{f}$ and the probability $P E R$ a packet is received in error, as a function of the packet size for both the cases of independent and burst errors under a high congested environment $(n=50)$. As expected, when the packet size becomes larger, both the error-collision probability and $P E R$ values increase, especially for high $B E R$ values. We note that $P E R$ attains similar values when we consider either independent $\left(B E R=10^{-6}\right)$ or burst errors (this is explained because in both cases we have similar $B E R$ values).

Figure 3 plots throughput efficiency and packet delay for the DIDD scheme by varying the number of stations under an error-prone environment. Although, DIDD decreases the chance of a packet collision by utilizing a higher contention window after a successful transmission instead of resetting it to $C W_{\text {min }}$, the larger the number of stations, the higher the error-collision probability and, thus, throughput and packet delay performance considerably degrades.

The previously derived performance results in [17]-[20] considered a fixed packet size. However, as figure 2 clearly depicts, the probability of a packet being in error highly depends on packet size apart from BER. Therefore, figures 4 and 5 examine the dependency of performance on the packet size by plotting the considered performance metrics versus $l$, for two different network sizes ( $n=5$ and 50). Note that for the case of independent errors, we consider two $B E R$ values $\left(B E R=10^{-4}\right.$ and $\left.10^{-6}\right)$. 
The 17th Annual IEEE International Symposium on Personal, Indoor and Mobile Radio Communications (PIMRC'06)

As has been shown in [8], throughput efficiency increases with increasing packet length in an ideal channel $(B E R=0)$. On the other hand, figure 4 illustrates that in an error-prone environment a trade-off exists between the desire to reduce the overhead by adopting a larger packet size and the need to reduce packet error rates by using smaller packet length. The figure clearly shows that there is a packet size that maximizes throughput performance in a heavily error-prone channel. This optimal packet length partly varies with the change of the number of contending stations but significantly depends on the $B E R$. In particular, in the case of good quality channel $\left(B E R<10^{-6}\right)$, excessive overhead in each packet actually limits the throughput; larger packet sizes improve throughput performance. As channel conditions deteriorate $\left(B E R=10^{-4}\right)$, it is better to employ a smaller packet size rather than a large one; the optimal packet length is approximately equal to 2000 bits for any network size. Conversely, we see that for large packet and network size values, packet delay considerably increases especially in high $B E R$ values.

Figure 5 illustrates the effect of packet payload size $(l)$ on throughput and packet delay performance for the employed Gilbert-Elliot burst error model. Note that in the Gilbert-Elliot model the $B E R$ in both the $G O O D$ and $B A D$ states is relatively low ( $10^{-10}$ and $10^{-5}$, respectively) but the average time spent on the two states is quite high. The figure depicts that the performance of the enhanced DIDD scheme is significantly sensitive to burst errors as well as to the utilized packet size. An interesting outcome is that the increase of network size plays an important role in attaining high packet delay values under a bursty error-prone environment.

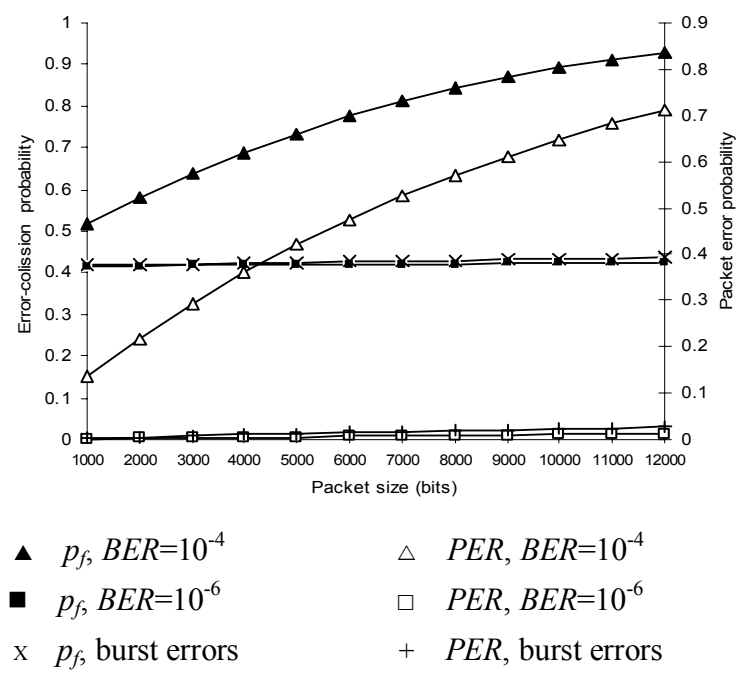

Figure 2 Error-collision probability and packet error probability versus packet size under independent and burst errors $(W=32, m=5, C=1 \mathrm{Mbit} / \mathrm{s}, n=50)$

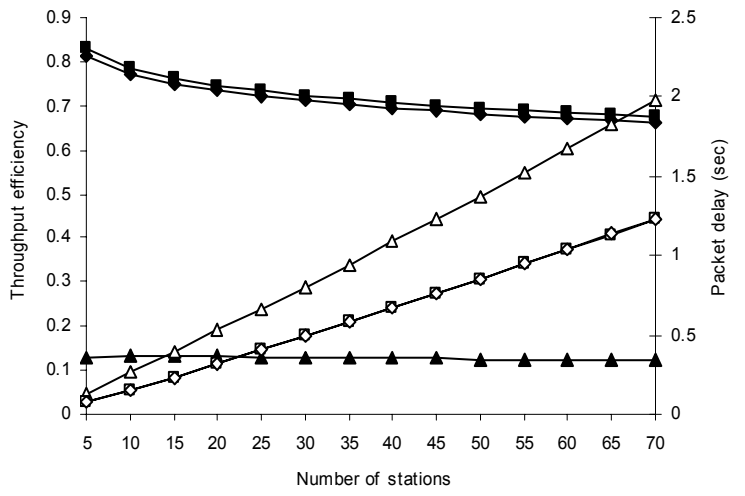
\ $S, B E R=10^{-4}$
$\triangle E[D], B E R=10^{-4}$
$S, B E R=10^{-6}$
$\square E[D], B E R=10^{-6}$
- $S$, burst errors
$\diamond E[D]$, burst errors

Figure 3 Throughput efficiency and packet delay versus number of stations under independent and burst errors ( $W=32, m=5, C=1 \mathrm{Mbit} / \mathrm{s}, l=1500$ bytes)

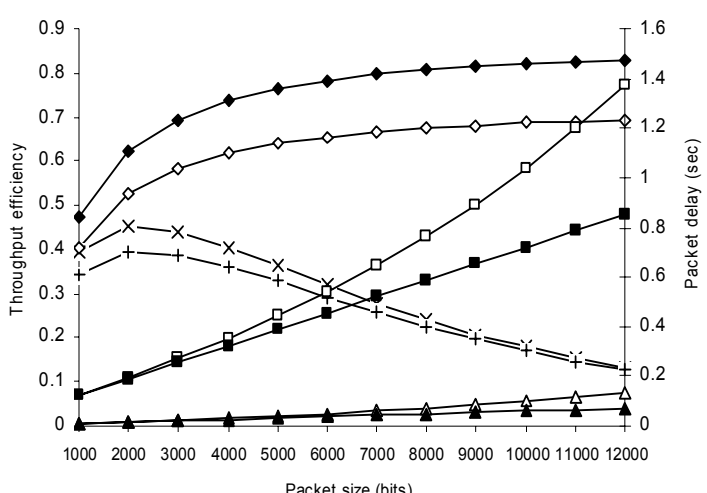
- $S, B E R=10^{-6}, n=5$
$\diamond S, B E R=10^{-6}, n=50$
X $S, B E R=10^{-4}, n=5$
$+\quad S, B E R=10^{-4}, n=50$
\ $E[D], B E R=10^{-6}, n=5$
$\triangle E[D], B E R=10^{-4}, n=5$
- $E[D], B E R=10^{-6}, n=50$
$\square \quad E[D], B E R=10^{-4}, n=50$

Figure 4 Throughput efficiency and packet delay versus packet size for various network sizes under independent errors $\left(W=32, m=5, C=1 \mathrm{Mbit} / \mathrm{s}, B E R=10^{-4}\right.$ and $10^{-6}$ )

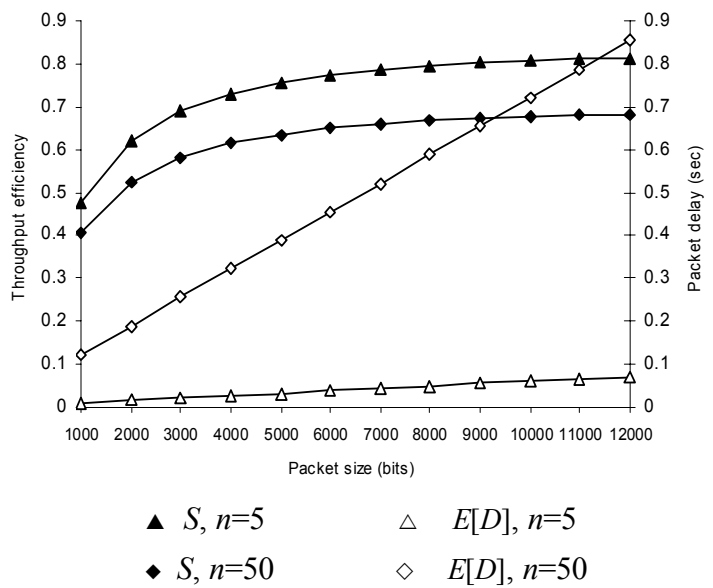

Figure 5 Throughput efficiency and packet delay versus packet size for various network sizes under burst errors for Gilbert-Elliot model $\left(W=32, m=5, C=1 \mathrm{Mbit} / \mathrm{s}, P_{g g}=0.97, P_{b b}=0.9, B E R_{g}=10^{-10}, B E R_{b}=10^{-5}\right.$ 
The 17th Annual IEEE International Symposium on Personal, Indoor and Mobile Radio Communications (PIMRC'06)

\section{Conclusions and Future Work}

In this paper, we introduced a mathematical analysis, which extends previous work by taking into account both independent and burst transmission errors for the DIDD backoff scheme. The proposed analysis derived simple mathematical expressions that calculate throughput efficiency and average packet delay. Analytical results illustrate that the DIDD scheme achieves a lower throughput and delay performance in fading conditions than in an ideal environment and was shown to be very sensitive to packet length. We have also proposed and studied performance under burst errors (when packet errors are correlated). The derived results show that the overall performance significantly depends on transmission errors and in particular on the time spent in the GOOD and $B A D$ states when burst errors are being considered.

Possible extensions of our work could include the study of time-varying bit error rate and burst errors under a larger variety of realistic scenarios as well as for non-saturated conditions.

\section{Acknowledgement}

This work is funded by the Greek Ministry of Education (25\%) and European Union (75\%) under the EPEAEK II program "Archimedes".

\section{REFERENCES}

[1] IEEE standard for Wireless LAN Medium Access Control (MAC) and Physical Layer (PHY) specifications, IEEE Std: 802.11b-1999/Cor 1-2001, 2001.

[2] G. Bianchi, "Performance Analysis of the IEEE 802.11 Distributed Coordination Function", IEEE Journal on Selected Area in Communications, vol.18, pp.535-547, 2000.

[3] H. Wu, Y. Peng, K. Long and J. Ma, "Performance of eliable Transport Protocol over IEEE 802.11 Wireless LAN: Analysis and Enhancement", in Proceedings of the IEEE INFOCOM,vol.2, pp. 599-607, 2002.

[4] P. Chatzimisios, A. C. Boucouvalas and V. Vitsas, "IEEE 802.11 Packet Delay - A Finite Retry Limit Analysis", in Proceedings of the IEEE Global Telecommunications Conference (Globecom 2003), vol. 2, pp. 950-954, San Francisco, USA, Dec. 2003.

[5] I. Vukovic and N. Smavatkul, "Saturation throughput analysis of different backoff algorithms in IEEE 802.11", in Proceedings of the IEEE Conference on Personal, Indoor and Mobile Radio Communications (PIMRC 2004), vol. 3, pp. 1870-1875, Barcelona, Spain, Sept. 2004.

[6] F. Cali, M. Conti and E. Gregori, "IEEE 802.11 protocol: Design and performance evaluation of an adaptive backoff mechanism," IEEE Journal on Selected Area in Communications, vol. 18, pp. 1774-1786, Sept. 2000.

[7] M. M. Carvalho and J. J. Garcia-Luna-Aceves, "Delay Analysis of IEEE 802.11 in Single-Hop Networks", in Proceedings of 11th IEEE International Conference on Network Protocols (ICNP 2003), pp. 146 - 155, 2003.

[8] P. Chatzimisios, "Performance Modelling and Enhancement of Wireless Communication Protocols", PhD thesis, School of Design, Engineering \& Computing (DEC), Bournemouth University, U.K., Dec. 2004.

[9] G. Bianchi and I. Tinnirello, "Remarks on IEEE 802.11 DCF Performance Analysis", IEEE Communication Letters, vol. 9, issue 8, pp. 765-767, Aug 2005.
[10] V. Vitsas, P. Chatzimisios, A. C. Boucouvalas, P. Raptis, K. Paparrizos and D. Kleftouris, "Enhancing performance of the IEEE 802.11 Distributed Coordination Function via packet bursting", invited paper, in Proceedings of the IEEE Globecom 2004 Workshops, pp. 245-252, USA, 2004.

[11] P. Chatzimisios, A. C. Boucouvalas and V. Vitsas, "Effectiveness of the RTS/CTS handshake in IEEE 802.11a Wireless LANs ", IEE Electronics Letters, vol. 40, issue 14, pp. 915 - 916, July 2004.

[12] P. Chatzimisios, A. C. Boucouvalas and V. Vitsas, "IEEE 802.11 Wireless LANs: Performance Analysis And Protocol Refinement", EURASIP Journal on Wireless Communications and Networking, vol. 2, pp. 67-78, 2005.

[13] M. Natkaniec and A. Pach, "An Analysis of Modified Backoff Mechanism in IEEE 802.11 Networks", in Proceedings of the Polish-German Teletraffic Symposium (PGTS 2000), pp. 89-96, Dresden, Germany, Sep. 2000.

[14] N.-O. Song, B.-J. Kwak, J. Song, and L. E. Miller, "Enhancement of IEEE 802.11 distributed coordination function with exponential increase exponential decrease backoff algorithm", in Proceedings of the 57th IEEE Semiannual Spring VTC, vol. 4, pp. 2775 - 2778, Apr. 2003.

[15] N. Qiang, I. Aad, C. Barakat and T. Turletti, "Modeling and analysis of slow CW decrease in IEEE 802.11", in Proceedings of the IEEE Conference Personal, Indoor Mobile Radio Communications (PIMRC 2003), pp. 17171721, Sept. 2003.

[16] B.P. Crow, I. Widjaja, J.G. Kim and P.T Sakai, "IEEE 802.11 wireless local area networks", IEEE Communications Magazine, vol. 35, no. 9, pp. 116-126, 1997.

[17] J. He, Z. Tang, Z. Yang, W. Cheng and C. Chou "Performance evaluation of distributed access scheme in error-prone channel", in Proceedings of the IEEE TENCON Conference, vol. 2, pp. 1142-1145, 2002.

[18] Z. Tang, Z. Yang, J. He and Y. Liu, "Impact of bit errors on the performance of DCF for wireless LAN", in Proceedings of the IEEE Conference on Communications, Circuits and Systems, vol. 1, pp. 529-533, 2002.

[19] Z. Hadzi-Velkov and B. Spasenovski, "Saturation throughput - delay analysis of IEEE 802.11 DCF in fading channel", in Proceedings of the IEEE International Conference on Communications (ICC 2003), vol. 26, pp. 121-126, 2003.

[20] P. Chatzimisios, A. C. Boucouvalas and V. Vitsas, "Performance analysis of IEEE 802.11 DCF in presence of transmission errors", in Proceedings of the IEEE International Conference on Communications (ICC 2004), vol. 7, pp. 3854-3858, June 2004.

[21] P.Chatzimisios, A. C. Boucouvalas, V. Vitsas, A. Vafiadis, A. Economidis and P. Huang "A simple and effective backoff scheme to enhance IEEE 802.11 MAC performance ", invited paper, in Proceedings of the $2^{\text {nd }}$ International Conference on Cybernetics and Information Technologies, Systems and Applications, vol. I, pp. 48-53, July 2005.

[22] E. N. Gilbert, "Capacity of a Burst-Noise Channel", Bell Systems Technical Journal, Vol. 39, pp. 1253-1266, Sept. 1960.

[23] M. Zorzi, R.R. Rao and L.B. Milstein, "Error statistics in data transmission over fading channels", in IEEE Transactions on Communcations, vol. 46, pp. 1468-77, 1998.

[24] Mauro Borgo, Andrea Zanella, Paola Bisaglia and Simone Merlin, "Analysis of the Hidden Terminal Effect in Multirate IEEE 802.11b Networks", in Proceedings of Wireless Personal Multimedia Communications (WPMC 2004), 2004. 